\title{
GMR Sensor Utilization for PCB Inspection Based on the Eddy-Current Testing Technique
}

\author{
K. Chomsuwan, Y. Fukuda, S. Yamada, M. Iwahara, H. Wakiwaka*, and S. Shoji* \\ Kanazawa University, 2-40-20 Kodatsuno, Kanazawa, Ishikawa 920-8667 \\ *Shinshu University, 4-17-1 Wakasato, Nagano 380-8553 \\ ${ }^{* *}$ TDK Corporation, 543, Saku, Nagano 385-8555
}

\begin{abstract}
This paper proposes the utilization of a GMR sensor as a magnetic sensor of an eddy-current testing probe for the detection of defects in printed circuit boards. The proposed probe consists of a meander coil functioning as an exciting coil and a GMR sensor functioning as a magnetic sensor. The GMR sensor has the advantages of high sensitivity to low magnetic fields over a broad range of frequency and very small size, and is therefore very useful for detecting very small cracks in printed circuit boards by means of the eddy-current testing technique. This paper describes the structure of the proposed probe, the inspection principles, and the experimental results achieved with an eddy-current testing probe using a GMR sensor in comparison with one using a solenoid coil sensor.
\end{abstract}

Key words: GMR sensor, solenoid coil, eddy-current testing, printed circuit board, inspection

\section{Introduction}

Normally, the identification of the defects on the printed circuit board (PCB) conductor is very difficult. Several techniques have been used for the PCB inspection. The optical method based on visual recognition processes is used however this technique is limited to inspect only on the outer surface of the PCB. Therefore, it is difficult to examine the small defects and disconnections occurred on the coated PCB conductor. The conductive tester, another technique, can inspect the conductor disconnections and short circuits, however this technique do not provide the information about partial defects on the PCB conductor. Moreover, the PCB conductor will obtain some mechanical stress from the contact actions.

Eddy-current testing (ECT) technique is a new method and very interesting for the PCB inspection because this is a non-contact testing method and can examine the conductor disconnections similar to an electrical testing approach. ECT probe, usually, consists of a pickup coil and an exciting coil and allows the detection of defects occurred on the coated PCB conductor. Many kinds of the pickup coil composed of multiple solenoid coils ${ }^{1), 2}$, figure of eight $\mathrm{coil}^{3)}$, and solenoid $\mathrm{coil}^{4)}$ were proposed to improve the characteristic of the PCB inspection based on ECT technique. Although the inspection results by the pickup coil as a magnetic sensor are achieved well, the identifications of the PCB defects are still complicated because of low output signals and limitation of the pickup

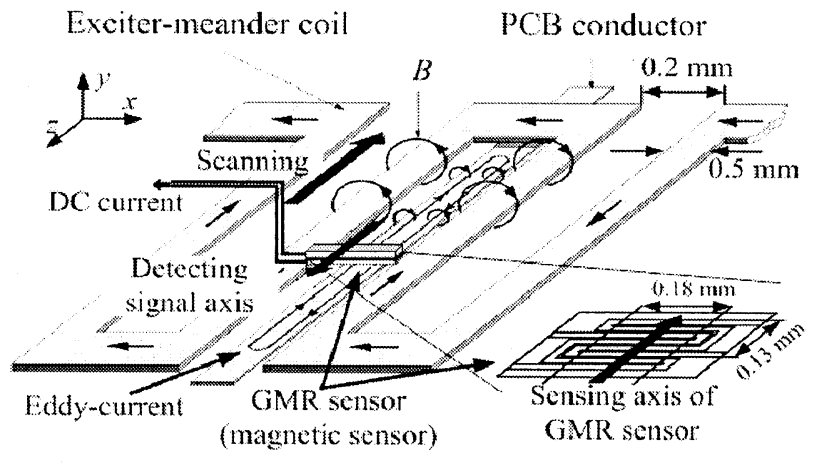

Fig. 1 Proposed eddy-current testing probe for PCB inspection.

coil such as dependence on frequency of output signal and difficulty in increasing spatial resolution.

Giant magneto-resistance (GMR) sensor is a magnetic sensor that, usually, is used as a magnetic read head in data storage technology. The resistance of the GMR sensor depends on varying of external magnetic field magnitude and the resistance is usually altered over $10 \%$ of the normal resistance. In addition, the GMR sensor has interesting features such as high sensitivity to low magnetic field over a broad range of frequency (from hertz to megahertz) especially in its sensing axis, high spatial resolution because of small size, and providing the precise and low noise output signal ${ }^{5}$. Furthermore, the GMR sensor can operate at room temperature and it is not difficult in fabrication the small size to enhance the spatial resolution. From the features above, the applying of the GMR sensor to the PCB inspection based on ECT technique will provide a good inspection technique for PCB conductor with less than $100 \mu \mathrm{m}$ in width.

In this paper, the utilization of the GMR sensor as a magnetic sensor of the ECT probe for the PCB inspection is proposed. The comparisons of the GMR sensor output signals with the solenoid coil output signals verify that this proposed probe is possible to be applied to the $\mathrm{PCB}$ inspection and provides an effective $\mathrm{PCB}$ inspection. Moreover, the PCB model inspection is also proposed.

\section{The proposed ECT probe construction}

\subsection{Basic ECT probe for $P C B$ inspection}

ECT probe for the PCB inspection, usually, consists of two parts as shown in Fig. 1. First is a meander coil which functions as an exciting coil. The meander coil generates the magnetic field which induces an eddy current in the 
PCB conductor. Any defect or boundary on the PCB conductor will effect to the eddy-current distribution in its conductor. Consequently, the magnetic field distribution will change as well. The magnetic sensor such as solenoid coil is a second part. Both normal and tangential components of the magnetic field, usually, will be detected by this solenoid coil. The tangential component, magnetic flux density in $z$-axis, occurs only at the defect points and the boundary of PCB conductor. Consequently, the output signals of the solenoid coil occurred at the defect or the soldering points will be proportional to the tangential component. Therefore, the identification of PCB defects can be performed by using the ECT technique.

\subsection{The proposed ECT probe with GMR sensor}

The GMR sensor as shown in Fig. 2 is used for this purpose. The voltage across the GMR sensor will fluctuate whenever the external magnetic field changes because the DC constant current was fed to the GMR sensor. The AC characteristics at each of GMR sensor axis are tested by using Helmholtz coil. From characteristic as shown in Fig. 3 , the GMR sensor has high sensitivity only to magnetic flux density in $z$-direction, $B_{z}$.

For the proposed ECT probe, the solenoid coil sensor is substituted by GMR sensor. From the characteristics above, the GMR sensor is attached to the meander coil as shown in Fig. 1 to detect only the tangential component. Therefore, the voltage across the GMR sensor will fluctuate when the defect points and the boundary of PCB conductor appear. Consequently, the principle of the proposed ECT probe with GMR sensor is still similarly to the principle of the ECT probe with the solenoid coil.

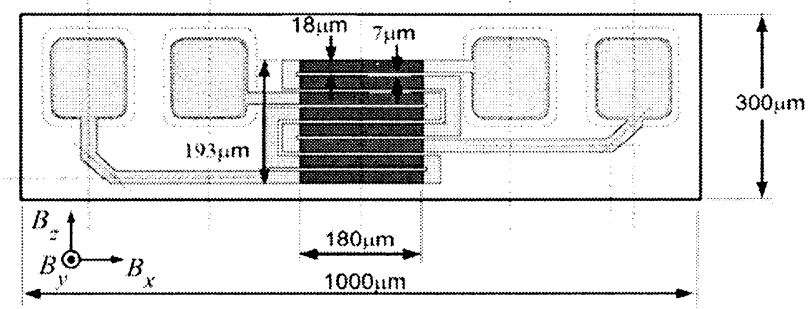

Fig. 2 GMR sensor element for PCB inspection.

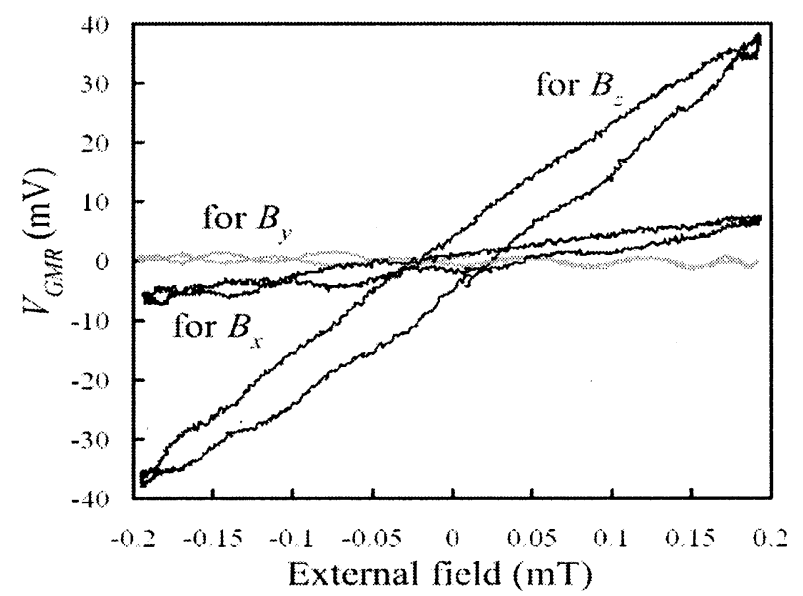

Fig. 3 AC characteristics of the GMR sensor for PCB inspection purpose at each of the GMR sensor axis.

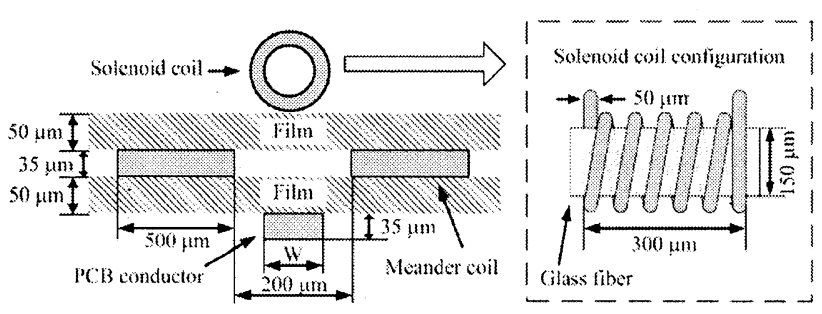

Fig. 4 Configuration of the probe with meander (exciting) solenoid coil (pick-up). Meander coil is sandwiched between films to fix the gap among solenoid coil and inspected $\mathrm{PCB}$ conductor.

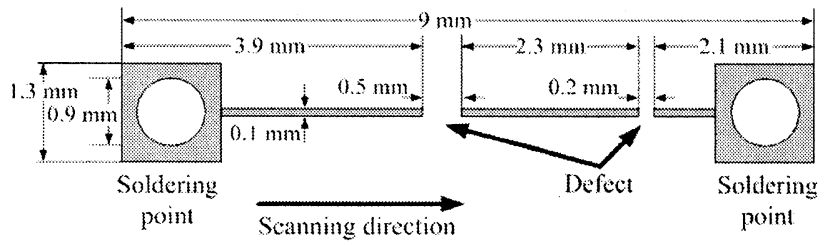

Fig. 5 Model of a PCB with defects and soldering points.
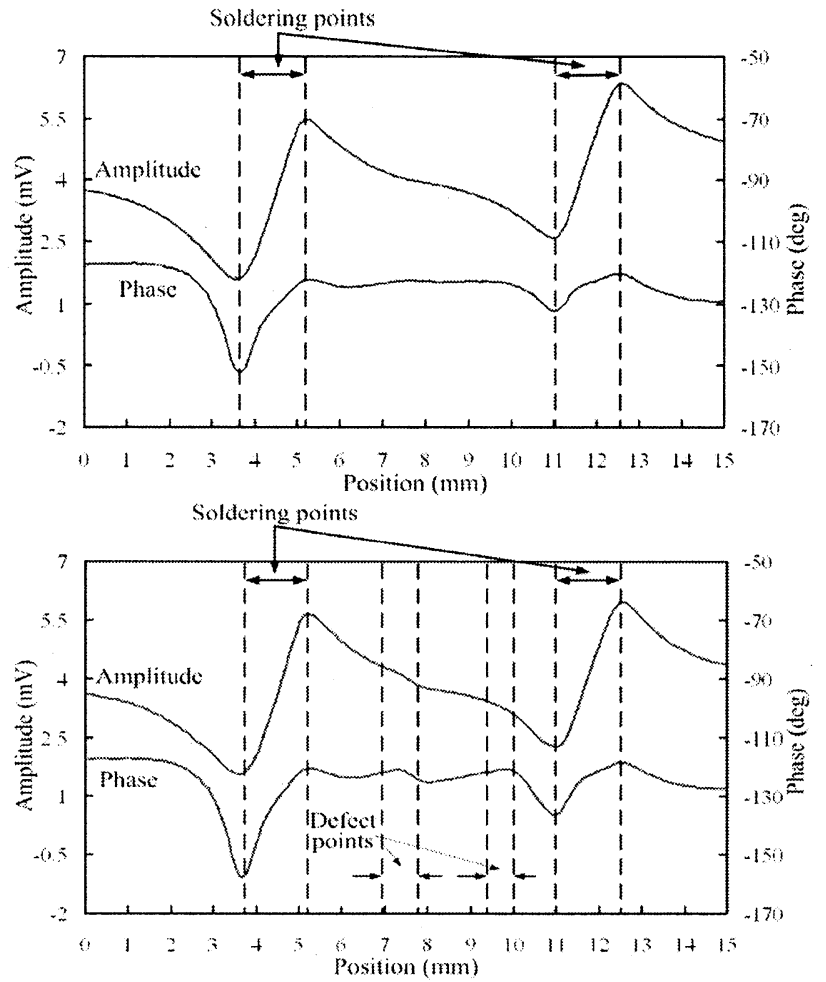

Fig. 6 Scanning signals of the non-defective PCB (top) and defective PCB (bottom), obtained with the solenoid coil sensor of the PCB model in Fig. 5.

\section{Experimental results}

\subsection{The configuration of system}

Sinusoidal current of $250 \mathrm{~mA}$ at frequency of $5 \mathrm{MHz}$ was fed to the meander coil. DC constant current of $5 \mathrm{~mA}$ was fed to the GMR sensor which has resistance of $631 \Omega$ when there is not any external magnetic field. The lock-in amplifier was used to measure an output signal changing obtained from the magnetic sensor. The scan pitch was set at $0.1 \mathrm{~mm}$. The experiment results obtained from the GMR 

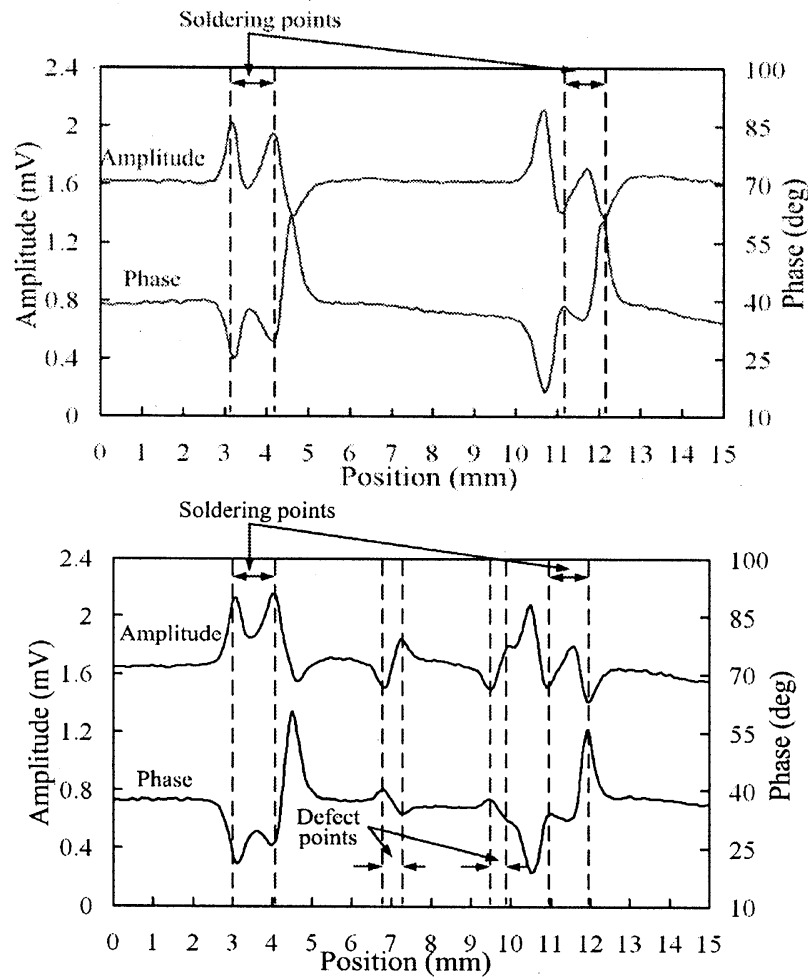

Fig. 7 Scanning signals of the non-defective PCB (top) and the defective PCB (bottom), obtained with the GMR sensor of the PCB model in Fig. 5.

sensor probe is compared with one obtained from the solenoid coil probe as shown in Fig. 4. The latter probe has a solenoid coil as pick-up sensor instead of GMR on the former

\subsection{One line PCB conductor inspection}

Fig. 5 shows one line PCB conductor model with defect and soldering points. This model was used to demonstrate the magnetic sensor output signals varying at the defect and soldering points.

Using solenoid coil probe, Fig. 6 shows detected signals that obtained from scanning the non-defect and defect PCB model shown in Fig. 5 respectively. Both of the output signals are compared to identify the defect points. Amplitude and phase output signals obtained from the solenoid coil vary at the defect and soldering points. The output signals have high variation at the soldering point whereas low varying output signals occur at the defect points. And the peaks of the output signals occur at the boundaries of the soldering point. Although the solenoid coil can detect the defect points, the identification of the defect points is very difficult because of the small output signal changing occurred at the defect points. In particular, the identification of defect points is more difficult whenever the defect points happen closer to the soldering points.

Fig. 7 shows the output signal of GMR sensor that achieved from scanning the PCB model shown in Fig. 5. In Fig. 7, the changing in magnitude of the amplitude and phase signals obtained from GMR sensor has occurred at defect and soldering points on the PCB conductors like the utilization of solenoid coil. And the output signal changing occurred at the defect points achieved from the GMR sensor provides clearer and more accurate altering than that from the solenoid coil sensor. Additionally, both amplitude and phase signals occurred at the soldering point can be identified holes of the soldering point. Therefore, 2-D PCB image generated GMR sensor output signals will provide detail which is similar to PCB model.

Normally, the output signals that achieved from both of the sensors contain a lot of noise and DC-offset. In the experiment, the numerical gradient technique is used to remove the DC-offset of the output signals and to enhance the output signals at the defect points because this technique is very simple and faster than other techniques such as the linear phase finite impulse response (FIR) digital filter. Fig. 8 shows the amplitude gradients of the GMR sensor and solenoid coil output signals obtained from scanning along PCB conductor, $100 \mu \mathrm{m}$ in width and $35 \mu \mathrm{m}$ in thickness, with conductor disconnection of 100 $\mu \mathrm{m}$. From Fig. 8, the amplitude gradients, $V_{s}$, derived from GMR sensor $(0.17 \mathrm{mV} / \mathrm{pitch})$ is around 2 times of that from the solenoid coil $(0.08 \mathrm{mV} / \mathrm{pitch})$ whereas the noise signal amplitude, $V_{n}$, is not different. The defect identification by the amplitude gradient signal derived from GMR sensor, therefore, is not difficult. In order to comparison of the output signals occurred at the defect

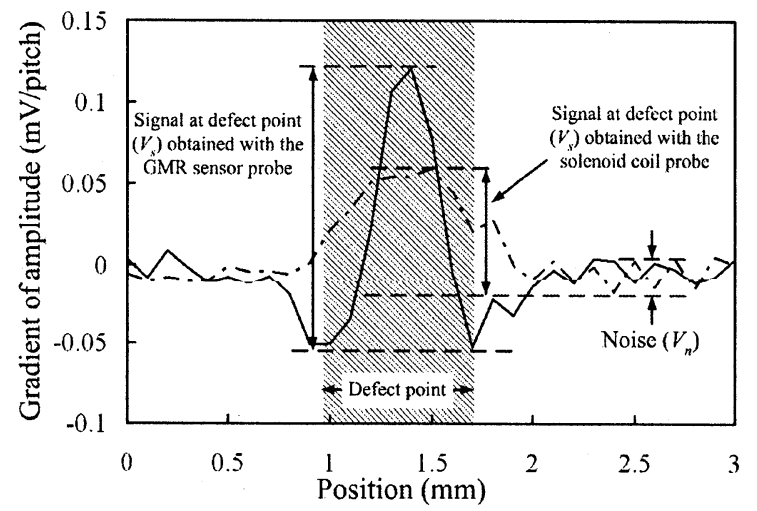

Fig. 8 Signal and noise amplitude gradients of the GMR sensor and solenoid coil.

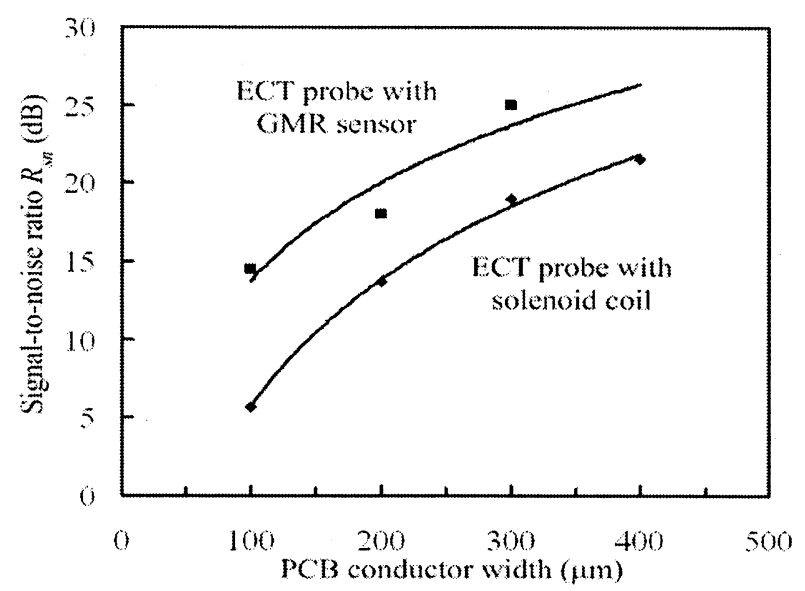

Fig. 9 Comparison of the signal-to-noise ratios of a GMR sensor and solenoid coil. 


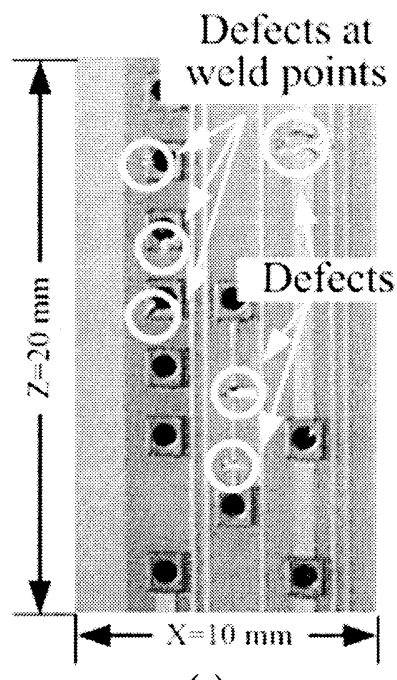

(a)

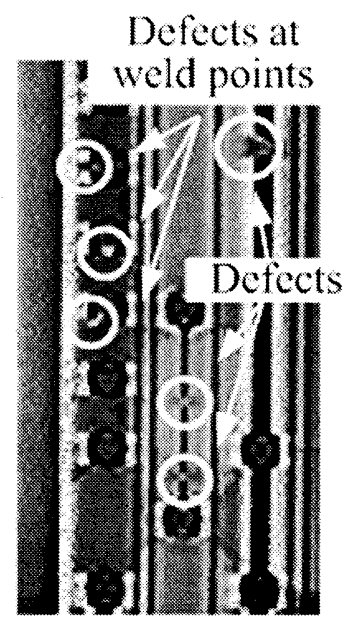

(b)

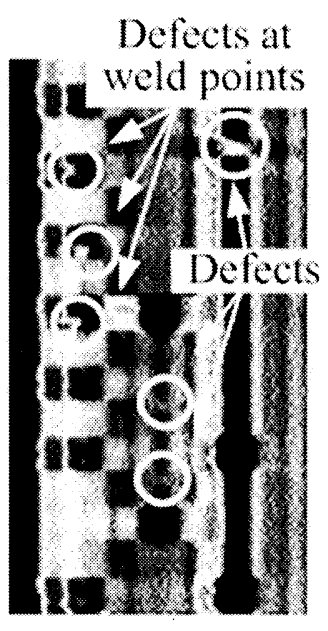

(c)

Fig. 10 (a) Photograph of the defective PCB model, (b) 2-D image obtained with a GMR sensor probe and (c) 2-D image obtained with a solenoid coil probe.

point, the signal-to-noise ratio, $R_{s n}$, is written by Eq.(1).

$$
R_{s n}=20 \log \left(V_{s} / V_{n}\right)
$$

Fig. 8 shows the comparison of the signal-to-noise ratios, $R_{s n}$, obtained from the GMR sensor probe and the solenoid coil probe. In application of the GMR sensor, the signal-to-noise ratios can be improved up to $8 \mathrm{~dB}$ approximately because the amplitude gradients, $V_{s}$, are improved as mentioned above. From the signal-to-noise ratios, $R_{s n}$, as shown in Fig. 9, the application of the solenoid coil probe for inspection of PCB conductor width that is less than $100 \mu \mathrm{m}$, high-density PCB inspection, is very difficult whereas the utilization of GMR sensor probe is possible. The reason is that the spatial resolution for GMR sensor probe is better than solenoid type according to the reduction of size. Moreover, the thickness of the GMR sensor effective area is around $100 \mathrm{~nm}$ therefore the GMR sensor is closer to PCB conductor than solenoid coil to detect the precise position of magnetic field variation.

\subsection{PCB model inspection}

The defect PCB model in Fig. 10 (a) was used in an experiment. The smallest size of the PCB conductor model is $100 \mu \mathrm{m}$. The defects on PCB conductor are classified into 2 types. First is the conductor disconnection on line conductor and second is the partial defect occurred at the soldering point. Fig. 10 (b) is a 2-D image achieved from the GMR sensor and Fig. 10 (c) is a 2-D image achieved from the solenoid coil. And defects on the PCB conductor are shown in white edge circles. The images from both the GMR sensor and the solenoid coil can be used to specify all of defects occurred on the PCB. The 2-D image achieved form GMR sensor provides the clear detail. Although the 2-D image achieved from solenoid coil can be used to specify the defects on PCB, the 2-D image has much noise that makes the image unclear. Therefore, the PCB defect specification using the 2-D image achieved from the GMR sensor is easier than that using the 2-D image achieved from the solenoid coil.

\section{Conclusion}

The utilization of a GMR sensor as an ECT probe improves an efficiency of the PCB inspection system based on ECT technique. Although the inspection results from the solenoid coil sensor can be achieved well, PCB image reconstruction is not clear enough to specify the defect points accurately. For the GMR sensor, PCB defects are identified easily because the GMR sensor probe provides higher output signal than the solenoid coil probe. Furthermore, the images from the GMR sensor signals provide higher quality than images from the solenoid coil signals.

Consequently, applying the PCB inspection based on ECT technique with GMR sensor with the high-density PCB is possible.

\section{Acknowledgment}

This work was supported in part by a Grand-in-Aid for Scientific Research from Japan Society for Promote of Science (Category B, 14350218).

\section{References}

1) D. Kacqrzak, T. Taniguchi, K. Nakamura, S. Yamada, and M. Iwahara: IEEE Trans. Magn., 37, 4, 2010 (2001).

2) K. Nakamura, S. Yamada and M. Iwahara:

The $15^{\text {th }}$ Symposium on Electromagnetic and Dynamics, 339 (2003).

3) T. Taniguchi, D. Kacqrzak, S. Yamada, and M. Iwahara: IEEE Trans. on Magnetics, 37, 4, 2790 (2001).

4) D. Kaeprzak, S. Yamada, and M. Iwahara, Insight, 42, 5, 312 (2000).

5) T. Dogaru and S. T. Smith, IEEE Trans. on Magnetics, 37, 5, 3831 (2001)

Received October 22, 2003; Accepted January 15, 2004 